\title{
283
}

\section{On Some Extensions of Bayesian Inference Proposed by Mr Lindley}

\author{
By R. A. FISHER \\ University of Adelaide
}

[Received October 1959. Revised January 1960]

SUMMARY

Critical comments are given on an extension of Bayesian inference proffered by Lindley $(1957,1958)$. The process is applied to the combination of two normal samples.

\section{INTRODUCTION}

IN 1956 the author developed a method of combining data of two distinct kinds, one portion being of a type capable of leading to an exact probability distribution by a fiducial argument, while the remainder, taken by itself, like the Bernouillian type of data considered by Bayes, leads to no statement of probability whatever. The procedure was essentially that of Bayes himself, using the first portion to obtain probabilities a priori while the second part, providing likelihood values only, supplies the multipliers by which the probabilities a priori are converted into logically similar probabilities a posteriori.

It had not occurred to me to consider whether this method might be applicable to the Combination of Observations in a more general sense, partly because the combination of observations of a similar kind presents no new problem (it must introduce the question of exhaustive estimation for the data as a whole, and not merely for one of its parts), partly also because Bayes himself did not discuss possible applications in this wider field.

In $1957 \mathrm{Mr} \mathrm{D}$. V. Lindley, who evidently disapproves of even the modest and restricted use I had made of Bayes's method, has perhaps inadvertently suggested a useful extension. In order to show that the method was erroneous (and overlooking even that the Section he was criticizing was headed "Observations of two kinds", the particular kinds intended being explained in the first paragraph), Lindley has applied Bayes's method boldly to some other problems of combination. He emphasizes that many of his results are contradictory and ridiculous, but this is doubtless due to his failure to recognize the problems to which the method may be correctly applied.

\section{AN EXAMPLE}

It may therefore be not out of place to exhibit an application of the process to the combination of two normal samples, using the fiducial distribution of the first sample to supply a simultaneous distribution of parameters $\mu$ and $\sigma$ a priori, and the second sample as the Bayesian observations used to modulate this prior distribution, and to give the distribution a posteriori.

From a sample yielding two statistics expressed explicitly as:

$$
\bar{x}_{1}=\frac{1}{N_{1}} S(x), \quad s_{1}^{2}=\frac{1}{N_{1}-1} S(x-\bar{x})^{2},
$$


the simultaneous fiducial distribution of the true mean, $\mu$, and the true standard deviation, $\sigma$, has been shown to be

$$
\frac{\sqrt{ } N_{1}}{\sigma \sqrt{(2 \pi)}} \exp \left[-\frac{N_{1}\left(\mu-\bar{x}_{1}\right)^{2}}{2 \sigma^{2}}\right] d \mu \times \frac{1}{\left(\frac{1}{2} N_{1}-\frac{3}{2}\right) !}\left(\frac{S_{1}}{2 \sigma^{2}}\right)^{\frac{1}{2} N_{2}-\frac{1}{2}} \exp \left[-\frac{S_{1}}{2 \sigma^{2}}\right] \frac{d \sigma^{2}}{\sigma^{2}}
$$

where $S_{1}$ stands for the sum of squares $\left(N_{1}-1\right) s_{1}^{2}$. In this the first factor specifies the distribution of $\mu$ in any array of the bivariate distribution having given $\sigma$, while the second factor gives the marginal distribution of $\sigma$, found by integrating out the value of $\mu$ in the first factor.

In (2) have been set out the frequencies expected, for given values of $\mu$ and $\sigma$, for the occurrence of a second sample of $N_{2}$ observations, having mean $\bar{x}_{2}$ within the range $d \vec{x}_{2}$, and sum of squares $S_{2}$ within the range $d S_{2}$;

$$
\frac{\sqrt{ } N_{2}}{\sigma \sqrt{(2 \pi)}} \exp \left[-\frac{N_{2}\left(\mu-\bar{x}_{2}\right)^{2}}{2 \sigma^{2}}\right] d \vec{x}_{2} \times \frac{1}{\left(\frac{1}{2} N_{2}-\frac{3}{2}\right) !}\left(\frac{S_{2}}{2 \sigma^{2}}\right)^{\frac{1}{2} N_{2}-\frac{3}{2}} \exp \left[-\frac{S_{2}}{2 \sigma^{2}}\right] \frac{d S_{2}}{2 \sigma^{2}} \text {. }
$$

The product of the four factors in (1) and (2), integrated both from $-\infty$ to $\infty$ with respect to $\mu$, and from 0 to $\infty$ with respect to $\sigma$, will supply a divisor by which the product as it stands may be reduced to a simultaneous distribution a posteriori.

Since only the two first factors involve $\mu$, the integration with respect to that variable may be carried out first, this operation being valid for and referring to each possible value of $\sigma$ in turn. The analysis will be simplified by writing

$$
\begin{gathered}
N_{1}+N_{2}=N, \quad N \bar{x}=N_{1} \bar{x}_{1}+N_{2} \bar{x}_{2}, \\
\frac{N_{1} N_{2}}{N}\left(\bar{x}_{1}-\bar{x}_{2}\right)^{2}+S_{1}+S_{2}=S .
\end{gathered}
$$

Now

$$
N_{1}\left(\mu-\bar{x}_{1}\right)^{2}+N_{2}\left(\mu-\bar{x}_{2}\right)^{2}=N(\mu-\bar{x})^{2}+\frac{N_{1} N_{2}}{N}\left(\bar{x}_{1}-\bar{x}_{2}\right)^{2}
$$

hence, on integrating with respect to $\mu$, there remains

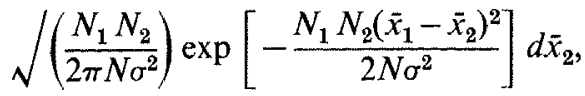

and, on dividing this into the product from which it was derived, the distribution a posteriori for $\mu$ in any array with assigned $\sigma$ is

$$
\frac{\sqrt{ } N}{\sigma \sqrt{(2 \pi)}} \exp \left[-\frac{N(\mu-\bar{x})^{2}}{2 \sigma^{2}}\right] d \mu \text {. }
$$

For the unconditional or marginal distribution of $\sigma$, independent of that obtained above since the integral of the latter is constantly equal to unity, we have first to integrate the product of the right-hand factors in (1) and (2) together with (3) from $\sigma=0$ to $\infty$. This gives

$$
\frac{\left(\frac{1}{2} N-\frac{3}{2}\right) !}{\left(\frac{1}{2} N_{1}-\frac{3}{2}\right) !\left(\frac{1}{2} N_{2}-\frac{3}{2}\right) !} \frac{S_{1}^{\left(\frac{1}{2} N_{1}-\frac{1}{2}\right)} S_{2}^{\left(\frac{1}{2} N_{\mathrm{s}}-\frac{3}{2}\right)} d S_{2}}{S^{\left(\frac{1}{2} N-\frac{1}{2}\right)}}
$$

and dividing the product by its normalizing factor, the integral (5) above, the probability of $\sigma$ lying in any defined range $d \sigma$ is

$$
\frac{1}{\left(\frac{1}{2} N-\frac{3}{2}\right) !}\left(\frac{S}{2 \sigma^{2}}\right)^{\frac{1}{2} N-\frac{1}{2}} \exp \left[-\frac{S}{2 \sigma^{2}}\right] \frac{d \sigma^{2}}{\sigma^{2}} \text {. }
$$


There is nothing essentially new in the distribution (4) and (6); it is only remarkable that the fiducial distribution can be inferred by applying Bayes's process to the combination of the two samples, and is identical with that derivable from a combination of the two bodies of data by methods familiar since the time of Gauss.

\section{Discussion}

Mr Lindley writes as though he had established important new limitations to the use of Bayes's method, but it should be remembered that he is not investigating the combination of data of two different kinds as carried out by Bayes, and discussed in my book. They are not in any sense limitations of the fiducial argument, but only of Lindley's proposed extension of the use of Bayes's argument. Whatever true limitations this may have they evidently do not exclude the simple application to normal data demonstrated above. It is not true, as Lindley (1957) asserts for normal distributions, that the argument is limited to families with known variance.

It is characteristic of the simultaneous fiducial distribution of the parameters of a normal population, that the distribution of the mean is not independent of that of the variance. It is, therefore, erroneous to base a Bayesian argument on prior information represented only by the marginal distribution of $\mu$, which is a distribution of $t$. Yates (1958) has called attention to the incompetence of this approach.

\section{REFERENCES}

Fisher, R. A. (1956), Statistical Methods and Scientific Inference. Edinburgh: Oliver \& Boyd. Chapter 5, Section 6.

LindLeY, D. V. (1957), "Review of Statistical Methods and Scientific Inference", Heredity, 11, $280-283$.

(1958), "Fiducial distributions and Bayes's theorem", J. R. Statist. Soc., B, 20, 102-107.

Yates, F. (1958), "Comments on Mr Lindley's review", Heredity, 12, 133-135. 